\title{
El juego como una estrategia didáctica para desarrollar el pensamiento numérico en las cuatro operaciones básicas*
}

\section{Game as didactic strategy to develop numerical thought in the four basic operations}

\author{
Jorge Hernán Aristizábal Z.** \\ Humberto Colorado T. *** \\ Heiller Gutiérrez Z****
}

\author{
**Magister en Educación, UCM. Profesor Universidad del \\ Quindío, Programa de Lic. en matemáticas, Colombia jhaz@ \\ uniquindio.edu.co.
}

***Magister en matemáticas, Magister en ciencias, especialidad en matemática Universidad UTP. Profesor Universidad del Quindío Programa de Lic. en matemáticas, Colombia colorado@uniquindio.edu.co

****Magister en Educación, UCM. Profesor Universidad del Quindío, Programa de Lic. en matemáticas, Colombia hgutierrez@uniquindio.edu.co

Cómo citar: Aristizabal, JH; Colorado H \& Gutierrez H. (2016) El juego como una estrategia didáctica para desarrollar el pensamiento numérico en las cuatro operaciones básicas. Sophia 12 (1): 117-125.

\section{Resumen}

Este artículo es el resultado de una investigación realizada por docentes pertenecientes al Grupo de Investigación en Educación Matemática de la Universidad del Quindío (GEMAUQ), en la cual se buscó desarrollar distintas habilidades y relaciones para familiarizarse y reforzar las operaciones básicas (adición, sustracción, producto y cociente) en estudiantes de grado quinto, asumiendo que el juego ocupa un lugar primordial entre las múltiples actividades del niño.La estrategia didáctica consistió en trabajar una serie de actividades y/o juegos en cada una de las operaciones matemáticas y la combinación de estas, al igual que en la resolución de problemas, cuya implementación permitió generar mayor motivación e interés en los estudiantes en el tema propuesto. Se ratifica, una vez más, que la enseñanza de las matemáticasutilizando el juego como una estrategia didáctica en reemplazo de los métodos didácticos convencionales aplicados en el aula de clase, logran la trasformación del proceso de enseñanza-aprendizaje y la forma en que docentes y estudiantes acceden al conocimiento en las cuatro operaciones básicas del pensamiento numérico.

Palabras Clave: Juegos matemáticos, estrategia didáctica, pensamiento numérico, operaciones básicas, educación matemática

*Este Artículo es resultado del proyecto de investigación: “ el juego como una estrategia didáctica para desarrollar el pensamiento numérico en las cuatro operaciones básicas”, desarrollado por tres docentes perteneciente al Grupo de Investigación en Educación Matemática de la Universidad del Quindío (GEMAUQ) 


\begin{abstract}
This article is the result of a research by teachers member of the Research Group of mathematical Education, del Quindio University (GEMAUQ), in which various skills and relationships were developed to familiarize and reinforce the basic operations, (addition, subtraction, product, and quotient), in students taking grade fifth, assuming that game plays a core role among the many activities performed by the child. The didactic strategy consisted of working a series of activities and/or games in each mathematic operation, and a combination of such activities, as well as in problem solving, which implementation led to improve and increase motivation and interest of students in the proposed topic. It is once again ratified that, teaching mathematics by using the game as a didactic strategy in replacement of conventional didactic methods in the classroom, transform the teaching-learning process, and how teachers and students achieve knowledge on the four basic operations of numerical thought.
\end{abstract}

Keywords: Mathematic games, didactic strategy, numerical thought, basic operations, mathematical education.

\section{Introducción}

El juego ocupa un lugar primordial entre las múltiples actividades del niño y en su desarrollo socio/ afectivo,en concordancia con Aristizábal, J; Colorado, H y Álvarez, D. (2011). Los cuales enuncian que "El juego como estrategia didáctica ycomo actividad lúdica en eldesarrollo integral del niño espertinente en el aprendizaje de lasmatemáticas, pues puede actuarcomo mediador entre un problemaconcreto y la matemática abstractadependiendo de la intencionalidad y el tipo de actividad..." (p.2), por tal motivo, el proyecto "El juego como una estrategia didáctica para desarrollar el pensamiento numérico en las cuatro operaciones básicas" permite desarrollar distintas habilidades de cálculo y relaciones, para familiarizarse y reafirmar el conocimiento de las operaciones básicas:adición, sustracción, producto y cociente, a través de la sorpresa, la práctica y la diversión, al igual que sirve de alternativa de evaluación dichas operacionespor su dinamismo, en los estudiantes degrado quinto.El juego como estrategia de enseñanza - aprendizaje ha mostrado resultados significativos en el aprendizaje de las matemáticas en los estudiantes; lo que se evidencia en algunas investigaciones como: "Investigación sobre juegos, interacción y construcción de conocimientos matemáticos", realizada por Eduardo Mercè $\mathrm{y}$ Deulofeu, Jordi del Departament de Didáctica de les Matemàtiques i les Ciències Experimentals de la Universidad Autónoma de Barcelona, "Eduardo Mercè y Deulofeu: aplicaciones prácticas para el aula infantil",realizada por Ruesga Ramos, María del Pilar.
La educación actual requiere de personas con capacidad crítica, analítica, reflexiva y esto se logra a través del desarrollo del pensamiento.Una persona con un desarrollo intelectual alto está capacitada para interpretar, argumentar, proponer, plantear y resolver problemas en diferentes contextos, por tanto, para la adquisición del sentido numérico es necesario proporcionar a los niños a través del juego situaciones ricas, variadas y significativas que estimulen la inteligencia e imaginación como lo plantean los estandares curriculares “... actividades centradas en la comprensión del uso y de los significados de los números y de la numeración; la comprensión del sentido y significado de las operaciones y de las relaciones entre números, y el desarrollo de diferentes técnicas de cálculo y estimación.” (p.58).

Por ello, los docentes de hoy tienen el reto de resignificar sus prácticas pedagógicas donde busquen que sus estudiantes se apropien de los conceptos y entiendan la importancia de las matemáticas. Al respecto, López (2005) afirma que: "se hace necesario buscar vías alternativas para la presentación de los contenidos a partir de situaciones y actividades que representen un sentido significativo para el alumno; estos permitirán a los estudiantes generar conjeturas, analizarlas con sus compañeros y poner en juego de manera consiente los conocimientos adquiridos con anterioridad".

El pensar involucra diferentes acciones mentales que evolucionan cuando el educando modifica sus estructuras cognitivas, pues el juego matemático en su dinámica pone en acción la capacidad para 
razonar, proponer, comunicarsede forma matemática desde la oralidad y la escritura; es decir, cuando se apropia del lenguaje, la historia, el significado de los conceptos matemáticos y la forma cómo estos involucran otros conceptos que a la vez se desarrollan en bucles generando cada vez un conocimiento con mayor solidez. Este proceso convierte al estudiante en el principal protagonista de su aprendizaje. El profesor Paulino Murillo (2003) afirma que el estudiante debe construir sus propios aprendizajes, que sean autónomos y que integren sus experiencias a otras ya conocidas para que no sigan en la búsqueda del desarrollo de la memoria y la repetición y es precisamente en éste tema donde se reconoce el avance del conocimiento adquirido.

El trabajo fue financiado por la Universidad del Quindío (Armenia, Colombia), ejecutado en la institución educativa Henry Marín Granada de Circasia, con niños y niñas degrado quinto; dicha investigación permitió el desarrollo del pensamiento numérico a través de la intervención del juego como estrategia para dominar las cuatro operaciones básicas, evidenciado la capacidad de relación, análisis, comprensión, procesos, abstracción, síntesis, generalización y en el desarrollo de los procesos que involucran las operaciones básicas.

\section{Materiales y métodos}

La investigación tuvo como objetivo principal diseñar y poner en funcionamiento una estrategia didáctica desde el juego, que fortalezca el pensamiento numérico en las cuatro operaciones básicas, en estudiantes de grado quinto, con la participación de la comunidad académica y del colectivo pedagógico. Para ello se realizó una investigación experimental, además exploratoria por cuanto sedesarrolló una estrategia didáctica que sirvió de ayuda a solucionardificultades encontradas en los estudiantes al abordar las operaciones básicas, en estudiantes de grado quinto lo que permite comprendercon mayor claridad la temática tratada.

La población objeto de estudio estuvo conformada por dos grupos de quinto grado del colegio Henry Marín Granada del municipio de Circasiaen el departamento del Quindío, con quienes se trabajó de acuerdo con el siguiente diseño experimental.

\section{Diseño experimental}

Se escogió el diseño experimentalpretest - postest tomando uno de los grupos como el grupo experimental y el otro como grupo control, es de notar que los grupos son noequivalentes debido a que tenían características similares pues, pertenecían a la misma institución, el mismo grado, a un estrato social equivalente y no habíaoscilaciones mayores entre sus edades.

Este diseño es denominado "diseño pretest - postest con un grupo control no equivalente" por Campbell y Stanley (1975) citado por Hernandez, Fernandez y Batista (2010) tiene la siguiente estructura:

La línea interrumpida indica que los grupos experimentales (E) y control (C) no han sido tomados al azar.

$$
\begin{aligned}
& O_{1} \text { y } O_{3}: \text { Son lasmediciones antes } \\
& O_{2} \text { y } O_{4}: \text { Son las mediciones después }
\end{aligned}
$$

\section{$X$ : Tratamiento}

En la investigación se sostiene la siguiente hipótesis: el desarrollo del pensamiento numérico en las cuatro operaciones básicas es mayor con el uso de una estrategia didáctica a través del juego que al utilizar una estrategia tradicional.

\section{Manipulación de la variable de investigación}

La variable a manipular fue la independiente: desarrollo del pensamiento numérico en las cuatro operaciones con las condiciones de estrategia didáctica y estrategia tradicional.

Los niños de los dos grupos, experimental y de control, responden a las pruebas pretest (mediciones antes del tratamiento) y postest (mediciones después del tratamiento).

\section{Tratamiento}

Los estudiantes del grupo experimental recibieron el tratamiento con la secuencia didáctica basado en actividades y juegos matemáticos al igual que la 
resolución de problemas. Es importante destacar que con la aplicación de la estrategia didáctica se potencian las operaciones mentales, que segun L. Alonso (2000) son:

- Receptivas: Observar, identificar y escuchar, Identificar elementos en un conjunto

- Comprensiva: analíticas (pensamiento analítico), comparar / relaciona, ordenar / clasificar, abstraer, resolución de problemas (pensamiento complejo), deducir / inferir, comparar / experimentar, analizar perspectivas / interpretar, transferir / generalizar, analizar / conectar, comprender / conceptualizar.

- Expresivas simbólicas: Representar (textual, gráfico, oral...) / Comunicar.

Los estudiantes del grupo control recibieron el tratamiento con la metodología tradicional. Según Flórez (1994), en el modelo tradicional el método básico del aprendizaje es el academicista, verbalista, que dicta sus clases bajo un régimen de disciplina a unos estudiantes receptores.

\section{Estrategia didáctica aplicada}

1. Se indagó mediante una entrevista a los docentes de grado quinto de la institución Henry Marín Granada del municipio de Circasia qué tipo de metodología utiliza en la enseñanza de las cuatro operaciones matemáticas (suma, resta, multiplicación y división).

2. De las seccionesde grado quinto de la institución se seleccionó al azar dos grupos como muestra; el grupo experimental y el de control.

3. Se aplicó al grupo control y al experimental el pretest para conocer los conceptos previos que tienen los estudiantes acerca de las operaciones básicas, las cualesel grupo de investigadores diseñó, validó $\mathrm{y}$, se aplicó al grupo de control como al experimental, este pretestconsta de un cuestionario de 16 preguntas, de las cuales 15 fueron enfocadas en una situaciónproblémica donde se involucraban una o varias operaciones, cuya respuesta era de selección múltiple con cuatro opciones de respuesta, pero con única respuesta, la pregunta restante eran ejercicios de desarrollo de operaciones matemáticas de forma algorítmica. El pretest tuvo como finalidad determinar el razonamiento que utilizan los estudiantes a la hora de resolver una situación problémica;de igual manera,se indagó por los conceptos previos y algoritmos que tenían acerca de operaciones de suma, resta, multiplicación y división.

4. Se estableció las condiciones de homogeneidad, para ello, se calificó los cuestionarios del pretest, se procesaron los resultados en elpaquete estadístico Statisticy se analizaron los resultados.

A continuación se presenta el análisis del pretest de los grupos control y experimental.

\section{Prueba de homogeneidad}

Figura 1. Análisis de los resultados del pretest

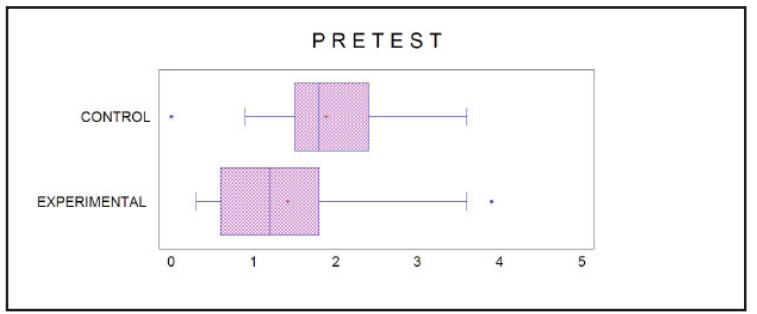

Fuente elaboración propia

En el diagrama de cajas se puede observar la homogeneidad de los resultados del pretest.El $50 \%$ de los estudiantes del grupo control obtuvo notas por debajo de 1,8 y $50 \%$ de los estudiantes del grupo experimental alcanzó notas por debajo de 1,2.

Figura 2. Comparación de los promedios del pretest

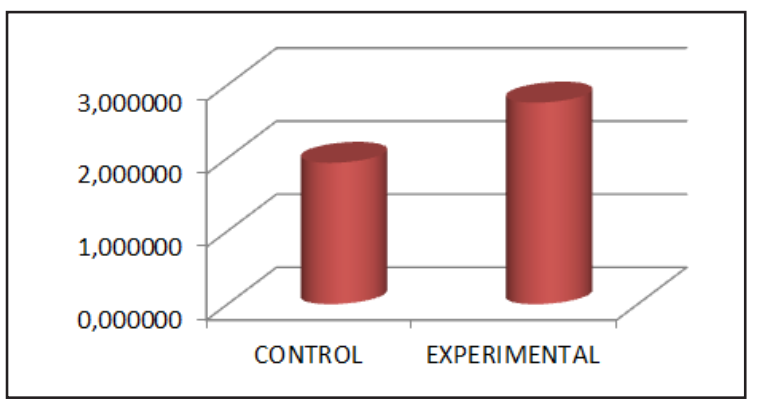

Fuente: Elaboración propia 
En la prueba $t$-studentrealizada para comparar los resultados del pretest del grupo control y del grupo experimental se observa que a un nivel de confianza del $95 \%$, no existe diferencia significativa entre los promedios de los grupos, es decir, los grupos son homogéneos. Como es evidencia con el P-value $(\mathrm{P}$-value $=0,124028>0,05)$. Lo anterior muestra que tanto el grupo control como el experimental obtuvieron resultados muy similares lo que indica que se encuentran a un mismo nivel de conocimiento del tema.

5. Se desarrolló en el grupo control, el tema de las operaciones básicas, siguiendo el modelo tradicional planteado por los docentes que dictan matemáticas en grado quinto la cual fue consultada en la entrevista previa.

6. Seimplementó en el grupo experimental el juego como una estrategia didáctica para desarrollar el pensamiento numérico en las cuatro operaciones básicas, la cual consistió en trabajar una serie de actividades y/o juegos que provocaron el interés y favorecieron la participación de los estudiantes en cada una de las etapas del juego utilizando operaciones matemáticas (suma, resta, multiplicación, división y la combinación de estas)al igual que en la resolución de problemas; aplicada al grupo experimental. Algunas de las actividades y/o juegos se realizaron en dos sesiones por la dinámica propia del juego.A continuación se presentan unas de las actividades que se utilizaron en el desarrollo de la estrategia didáctica.

Figura 3. Actividad de la estrategia utilizando el Tangram

Asumiendo que el triángulo más pequeño vale 1

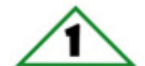

cuánto vale el tamgram completo?

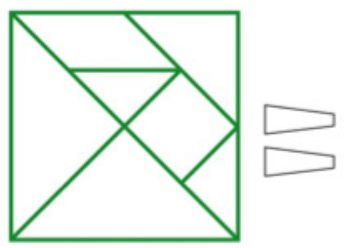

Cuánto vale cada una de las siguientes figuras
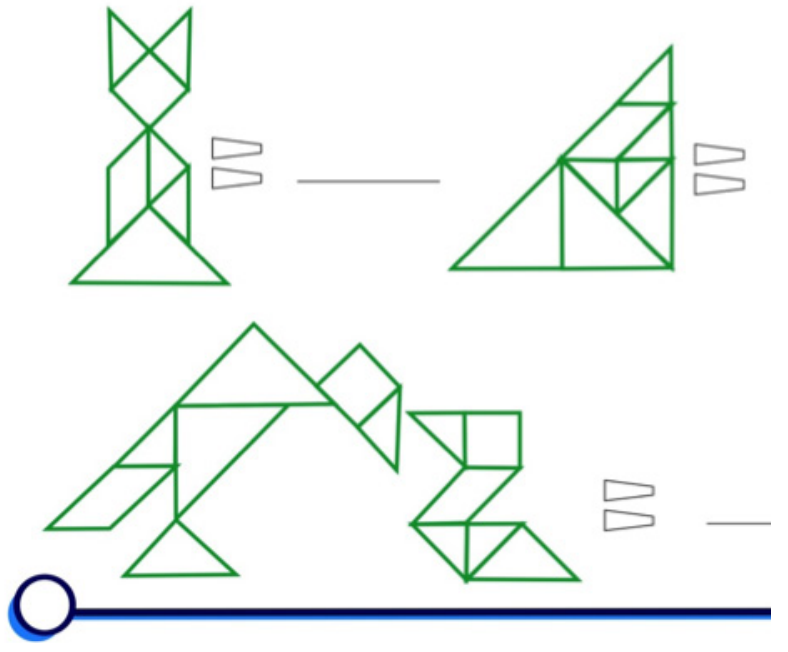

Figura 4. Actividad de la estrategia utilizando actividades escritas

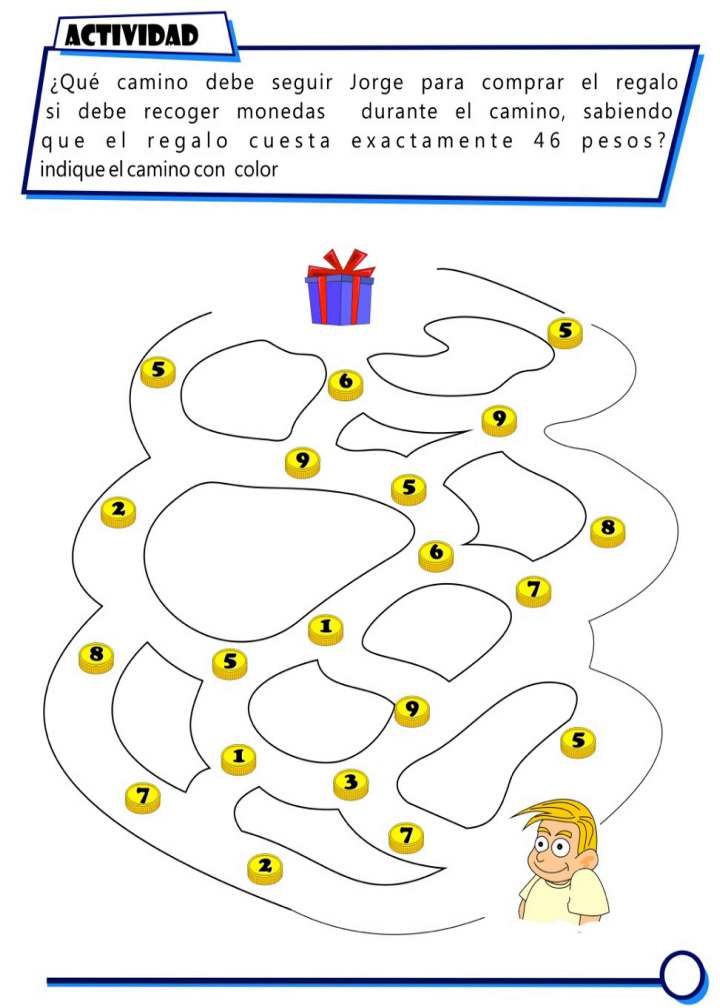

Fuente: Elaboración propia 
Figura 5. Actividad de la estrategia utilizando juego diseñado

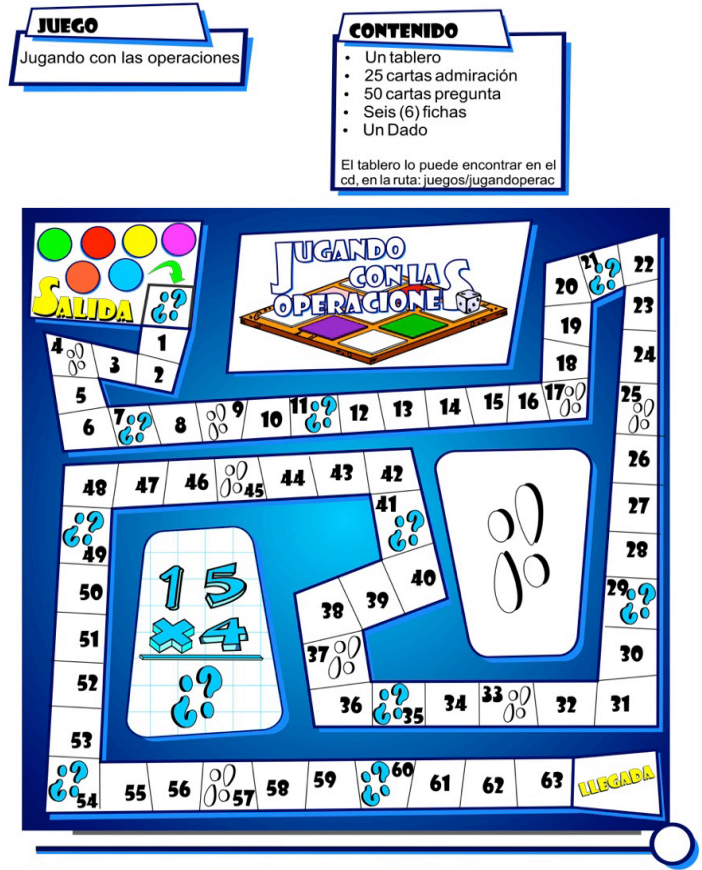

Fuente: Elaboración propia

7. Durante el trascurso del proceso investigativo se realizó seguimiento continuo, un constante monitoreo a los grupos de la muestra, para determinar las actitudes que tomaban los estudiantes frente a las metodologías, en la mayoría de los casos este monitoreo se obtuvo a través de observación participante.

8. Terminada la estrategia se aplicó un postest (el cual consistía en el mismo cuestionario pretest), a los grupos que conformaban la muestra.

A continuación se presentan los cálculos de la media aritmética, mediana y desviación estándarde los grupos del pretest y postest. pag 123.

Tabla 1. Comparación de lamedia, mediana y desviación estándar entre pretest y postest

\begin{tabular}{|c|c|c|c|c|c|c|}
\hline \multirow{2}{*}{ Grupo } & \multicolumn{2}{|c|}{ Media } & \multicolumn{2}{|c|}{ Mediana } & \multicolumn{2}{|c|}{ Desviación estándar } \\
\hline & Pretest & Postest & Pretest & Postest & Pretest & Postest \\
\hline Control & 1,8833 & 1,93333 & 1,8 & 1,8 & 0,82836 & 0,79187 \\
\hline Experimental & 1,4143 & 2,75714 & 1,2 & 3 & 1,00463 & 0,951615 \\
\hline
\end{tabular}

\section{Resultados}

Técnicas de análisis estadístico de resultados: de acuerdo con el diseño experimental se aplicó la prueba $t$-studentpara establecer las diferencias entre los grupos comparados, de los cuales se muestra el análisis del postest de los grupos control y experimental.

Figura 6. Análisis de los resultados del postest

\section{POSTEST}

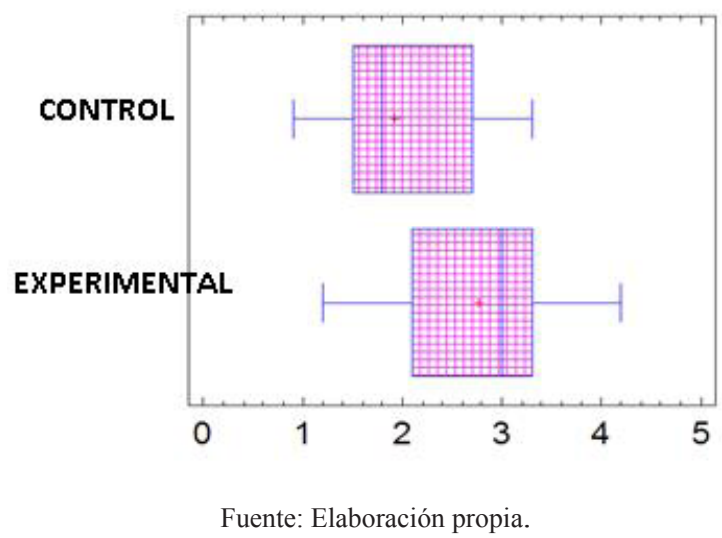

Figura 7. Comparación de los promedios del postest

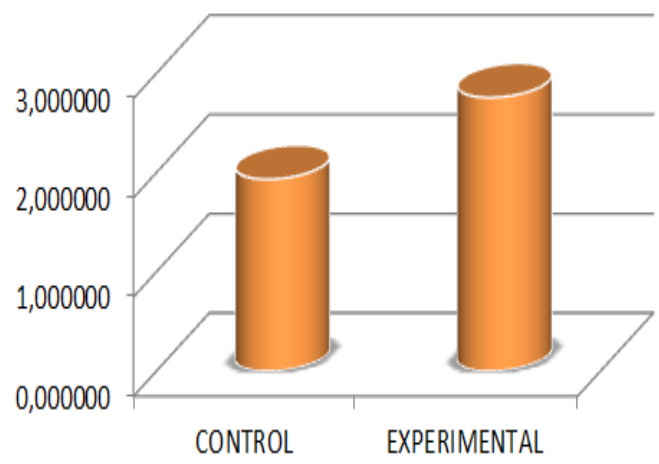

Fuente: Elaboración propia

Tabulación e interpretación de los resultados obtenidos: al comparar la media, la mediana y la desviación estándar entre el pretest y postest(tabla 1)se evidencia una mayor eficiencia en el grupo experimental al utilizar la estrategia del juego 
referente al grupo control con la metodología tradicionalutilizada por el docente, en el diagrama de cajas (figura 6) se puede observar la heterogeneidad de los resultados del postest.E1 $50 \%$ de los estudiantes del grupo control obtuvo notas por debajo de 1,8 y 50 $\%$ de los estudiantes del grupo experimental obtuvo notas por debajo de 3,0 .

En la prueba t-student realizada para comparar los resultados del postest del grupo control y del experimental (figura 7) se observa que a un nivel de confianza del $95 \%$, existe diferencia significativa entre los promedios de los grupos. (P-value $=$ $0,00611012<0,05$ )

\section{Discusión de resultados}

Los psicólogos destacan la importancia del juego en la infancia como medio de formar la personalidad $\mathrm{y}$ de aprender de forma experimental a relacionarse en sociedad, a resolver problemas y situaciones conflictivas. En el trabajo realizado se pudo apreciar que la aplicación de los diferentes juegos desarrollados en la estrategia, propiciaron en los estudiantes situaciones de integración, interacción, liderazgo, confrontación de ideas y generación de estrategias para dar resolución a los problemas o desafíos planteados en los juegos; todo ello permitió la apropiación de conceptos y desarrollo de pensamiento numérico. Según Ogalde y Bardavid (1997), las ventajas que aportan los materiales didácticos los hacen instrumentos indispensables en la formación académica: Proporcionan información y guían el aprendizaje, es decir, aportan una base concreta para el pensamiento conceptual y contribuye en el aumento de los significados; desarrollan la continuidad de pensamiento, hace que el aprendizaje sea más duradero y brindan una experiencia real que estimula, la actividad de los estudiantes.

Los cinco procesos generales que se establecen en los Lineamientos curriculares de matemáticasson formular y resolver problemas; modelar procesos y fenómenos de la realidad; comunicar; razonar y comparar, ejercitar procedimientos y algoritmos. En los diferentes momentos vividos en el desarrollo de la estrategia, se generaron espacios donde los estudiantes a través del juego pudieron acercarse a los algoritmos de las operaciones con números naturales mejorando su habilidad de cálculo mental.
Posteriormente, se trabajaron situaciones problemas cuya solución se buscaba a través de los juegos, identificando cuales operaciones debían tener en cuenta para encontrar dicha solución. Al respecto Godino (2004) plantea que a través de la resolución de problemas matemáticos, los estudiantes deberán adquirir modos de pensamiento adecuados, hábitos de persistencia, curiosidad y confianza ante situaciones no familiares que les serán útiles fuera de la clase de matemáticas.

También Jean Piaget (1978) estudió la transición de la manera de razonar de los adolescentes de lo que él llamó "el pensamiento operatorio concreto" al "operatorio formal" y propuso un conjunto de operaciones lógico-matemáticas que podrían explicar ese paso. Cuando los estudiantes manipularon los diferentes juegos propuestos en la estrategia, se evidenciaba un pensamiento concreto, el cual después de varias sesiones, permitió en algunos estudiantes hacer transición de pensamiento concreto a pensamiento formal.

Por otra parte, Miguel de Guzmán (1992), señala que, más allá de las ramas tradicionales de las matemáticas: la aritmética y la geometría, en su devenir histórico el espíritu matemático habría de enfrentarse con los siguientes aspectos:

-La complejidad del símbolo.

-La complejidad del cambio y de la causalidad determinística.

- La complejidad proveniente de la incertidumbre en la causalidad múltiple incontrolable.

-La complejidad de la estructura formal del pensamiento.

Aquí se puede ver una clara relación con los cinco tipos de pensamiento matemático enunciados en los Lineamientos curriculares: en la aritmética, el pensamiento numérico; en la geometría, el pensamiento espacial y el métrico; en el álgebra y el cálculo, el pensamiento métrico y el variacional, en la probabilidad y estadística, así como en el pensamiento aleatorio. En la estrategia didáctica aplicada en el proyecto se desarrolló el pensamiento numérico a través del juego, en las cuatro operaciones 
básicas soportadas en los demás pensamientos, lo cual da cabida a generar nuevas investigaciones en los otros pensamientos a través del juego como estrategia didáctica.

Para validar la apropiación de los conceptos se trabajo con situaciones problémicas, cuya solución requería de las operaciones básicas (adición, sustracción, producto y cociente) con lo que se mostró la aplicabilidad de los temas vistos y la secuencia lógica para llegar a la solución del problema. Según Vergnaud (1995): la enseñanza por resolución de problemas pone el énfasis en los procesos de pensamiento, en los procesos de aprendizaje y toma de contenidos matemáticos, considerando importante que el estudiante manipule los objetos matemáticos, active su propia capacidad mental, y ejercite su creatividad, reflexione sobre su propio proceso de pensamiento, haga transferencia de actividades $\mathrm{u}$ otros aspectos de su trabajo mental, que adquiera confianza en sí mismo, que se divierta con su propia actividad mental, que se prepare para otros problemas de la ciencia y de la vida cotidiana y para los nuevos retos de la tecnología y la ciencia.

Se pudo evidenciar que la poca comprensión lectora en ambos grupos fue un factor determinante en las dificultades para la resolución de problemas, por lo que es de vital importancia que los docentes brinden a los estudiantes las herramientas necesarias para mejorar la comprensión e interpretación de situaciones problémicas. Parra (1990) afirma que:

La resolución de problemas se analiza delimitada a situaciones de aprendizaje intencionalmente estructuradas y vinculadas con algún campo de estudio, como las que se dan en la dinámica escolar, ese disponer de los elementos para comprender la situación que el problema describe, supone que el sujeto que habrá de resolver el problema en cuestión, ha tenido acceso o ha construido aquel conocimiento declarativo $y$ el respectivo conocimiento procedimental que son requeridos como antecedente mínimo necesario para poder comprender información, establecer relaciones y utilizar procedimientos con la finalidad de llegar a resolver el problema que se le ha planteado.

\section{Conclusiones}

El objetivo general se cumplió a través de la implementación de la estrategia didáctica desde el juego, ya que permitió fortalecer el pensamiento numérico en las cuatro operaciones básicas, en los estudiantes de grado quinto de la Institución Educativa Henry Marín Granada del municipio de Circasia en el departamento del Quindío.Los grupos que ingresaron tenían condiciones homogéneas, es decir, tenían las mismas características en términos de los presupuestos teóricos con que cuentan al inicio de la ejecución del proyecto de investigación, lo que permitió mayor eficiencia en las pruebas $\mathrm{y}$ actividades que se realizaron para alcanzar los objetivos propuestos.

La implementación del juego permitió generar mayor motivación e interés en los estudiantes en el tema propuesto.Se comprobó la hipótesis de trabajo, pues se evidenciaron diferencias significativas en los puntajes registrados en el pretest y el postest de los grupos, tanto de control y como del experimental.

En una didáctica como la desarrollada en este proyecto, la matemática adquiere un nuevo significado para el estudiante. Además de la motivación y buena actitud que mostraron los estudiantes al trabajar con juegos, se resaltan otros aspectos importantes a los procedimientos simplemente algoritmos como los relacionados con el dar resultados y sin la argumentación.

Los juegos grupales fueron de mayor acogida por los estudiantes pues permitía generar competencia entre ellos.En estos momentos de cambio, se hace necesario reflexionar en la enseñanza de las matemáticas, en cuanto a los métodos didácticos convencionales utilizados en el aula de clase, y procurar por la trasformación del proceso de enseñanza-aprendizaje y la forma en que docentes y estudiantes acceden al conocimiento.

\section{Recomendaciones}

Teniendo en cuenta la realidad educativa, se recomienda a los docentes plantear y acoger estrategias pedagógicas y didácticas innovadoras en el marco del juego como estrategia de enseñanza, que conlleven al desarrollo del pensamiento matemático. Se sugiere 
dar continuidad a la propuesta del Juego como una estrategia didáctica para desarrollar el pensamiento numérico en las cuatro operaciones básicas y en otros temas, como una estrategia eficaz para superar las dificultades encontradas en la educación matemática.

Se sugiere a los docentes del área de matemáticas de educación básica, la aplicación de las estrategias orientadas a desarrollar el pensamiento matemático en los estudiantes, para potenciar las habilidades que les permitan mejorar el acceso al saber.

Las actividades desarrolladas en este proyecto muestran el arduo proceso que acarrea elaborar los juegos y las actividades propuestas, pero este esfuerzo será en vano en la medida en que las instituciones y los docentes no involucren estos materiales en su aula de clase.

Ante esta situación la universidad se debe convertir en un eje articulador para la construcción de materiales educativos para las instituciones de educación básica y media. Esto permitirá a largo plazo el desarrollo de estrategias propias de la región.

\section{Referencias bibliográficas}

Alonso, L. (2000). ¿Cuál es el nivel o dificultad de la enseñanza que se está exigiendo en la aplicación del nuevo sistema educativo?. Revista educar. 26, 53-74.

Aristizabal, J; Colorado, H y álvarez, D. (2011). El juego en el desarrollo del pensamiento: numérico, las cuatro operaciones. Armenia. Elizcom.

Campbell, D T y Stanley, J. C. (1975). Diseños experimentales y cuasi-experimentales en la investigación social. Amorrotu.

Edo, M. y deulofeu, J. (2006). Investigación sobre juegos, interacción y construcción de conocimientos matemáticos. Departament de Didáctica de les Matemátiques i les CienciesExperimentals. Universitat Autónoma de Barcelona. Disponible en http://www. raco.cat/index.php/Ensenanza/article/ view/75830
Flórez, R. (1994) Hacia una pedagogía del conocimiento.Bogotá: McGraw Hill, .

Godino, J. et al (2004), “Didáctica de las matemáticas para docentes". Proyecto Edumat Docentes. Universidad de Granada.

Guzmán, M. (1992): Tendències innovadores en educaciómatemàtica. Butlletí de la Societat Catalana de Matemàtiques, 7, 7-33. Barcelona

Hernandez, R. Fernandez, C. Baptista P. (2010). Metodología de la Investigación. México: Mc-Graw Hill.

Ministerio de educación nacional (2006). Estándares básicos de competencias en matemáticas. Bogotá: M.E.N. .

Murillo P. (2003). Qué es el aprendizaje significativo y cuál es su importancia en el aprendizaje de la matemática. Disponible en: http:// www.utp.ac.pa/articulos/aprendizaje/ significativo.htm.

Ogalde, I. Bardavid, E. (1997). Los materiales didácticos. Medios y recursos de apoyo a la docencia. México: Trillas

Parra, B. (1990). "Dos concepciones de resolución de problemas", Revista Educación Matemática, 2(3),22-31.

Piaget, J. (1978). Introducción a la epistemología genética I. El pensamiento matemático (2a. ed.). Buenos Aires: Paidós.

Vergnaud, G. (1995). El niño, las matemáticas y la realidad. México: Trillas. . 



\section{Lo bello y lo bueno en el discurso oral y escrito: Implicaciones pedagógicas del Fedro de Platón*}

\section{The beautiful and the good in oral and written speech: Pedagogic implications of Platón Phaedrus}

\author{
Carlos Adolfo Rengifo Castañeda ** \\ Diana Carolina Cañaveral *** \\ Mario Alberto Álvarez López **** \\ Luz Adriana Bernal López *****
}

\begin{abstract}
** Doctorando en Filosofía de la Universidad Pontifica Bolivariana de Medellín. integrante de los grupos de investigación Paideia de la UGCA y Educación: Desarrollo Humano de la USB de Cali. Docente de la Universidad San Buenaventura de Cali. e-mail: careca1106@ gmail.com y carengifo1@usbcali.educo.
\end{abstract}

***(E) Mg. en Derecho Público. Docente investigadora, líder del Grupo de Investigación de Derecho Público de la Universidad la Gran Colombia Seccional Armenia. E-mail: canaverallondiana@miugca. edu.co.

**** PhD. en Ciencias de la Educación. Investigador del Grupo de Investigación Educación y Desarrollo Humano, Universidad San Buenaventura Cali. Email: marioalbertoal@gmail.com y maalvarez2@usbcali.edu.co.

*****Licenciada en Biología y Educación Ambiental de la Universidad del Quindío, Profesional en Gestión de Recursos Naturales, docente universitaria e-mail: luzadrib29@gmail.com.

Cómo citar: Rengifo, C.A; Cañaveral, D.C; Álvarez, M.A \& Bernal, L.A. (2016) Lo bello y lo bueno en el discurso oral y escrito: Implicaciones pedagógicas del fedro de Platon.Sophia 12(1):127-137.

\section{Resumen}

Este artículo pretende comprender los argumentos expuestos por Platón en el diálogo del Fedro, que permiten identificar algunas de las implicaciones de su propuesta en el discurso tanto oral como escrito y su incidencia en el pensamiento contemporáneo. Lo anterior, mediante un ejercicio de revisión bibliográfica (apelando a fuentes primarias y secundarias), que posibilitan el reconocimiento de que, finalmente, solo quien proceda del modo señalado por este discípulo de Sócrates en el texto del Fedro, podrá ser llamado "filósofo, amante de la sabiduría".

Palabras Clave: Filosofía, pluralismo, oratoria, discurso, pedagogía, fedro. 